\title{
The characteristics of keratomycosis by Beauveria bassiana and its successful treatment with antimycotic agents
}

\author{
Hiroko Sonoyama' \\ Kaoru Araki-Sasaki' \\ Shigeyasu Kazama' \\ Tsutomu Kawasaki' \\ Hidenao Ideta' \\ Atsuko Sunada ${ }^{2}$ \\ Seishi Asari² \\ Yoshitsugu Inoue ${ }^{3}$ \\ Kozaburo Hayashi ${ }^{4}$ \\ 'Ideta Eye Hospital, Kumamoto, \\ Kumamoto, Japan; ${ }^{2}$ Department of \\ Laboratory for Clinical Investigation, \\ Osaka University Hospital, \\ Suita, Osaka, Japan; ${ }^{3}$ Division of \\ Ophthalmology and Visual Science, \\ Department of Medicine of Sensory \\ and Motor Organ, Tottori University, \\ Yonago, Tottori, Japan; ${ }^{4}$ mmunology \\ and Virology Section Lab, Immunology, \\ NEI, NIH, Bethesda, MD, USA
}

Correspondence: Kaoru Araki-Sasak Ideta Eye Hospital, I-35 Gofuku-cho, Kumamoto City, Kumamoto 8600035 , Japan

Tel +81963255222

Fax +81963115512

Email sasakis@sa2.so-net.ne.jp

\begin{abstract}
Clinical findings and treatment of keratomycosis caused by Beauveria bassiana, an entomopathogenic filamentous fungus, are described for an 80-year-old woman, who was referred to the hospital for ocular pain and redness on the 9th day after an ocular injury caused by the frame of her glasses. She had a long history of recurrent diabetic iritis and continuously used topical antibiotics and corticosteroids. At her first visit, a slit-lamp examination indicated a corneal ulcer confined within the superficial stromal layer, along with a slight infiltration and edema. Only a very few inflammatory cells were seen in the anterior chamber. Direct microscopic examination of corneal scrapings revealed septate fungal hyphae with zig-zag rachis and budding that was subsequently identified as $B$. bassiana by slide culture. Topical voriconazole with miconazole, pimaricin and oral itraconazole were effective and the lesion disappeared leaving only a mild scar at 2 months. The sensitivity of $B$. bassiana to various antimycotic agents was confirmed by broth microdilution, agar dilution with the Clinical Laboratory Standard Institute standard, and a disk method using topically applied concentrations. B. bassiana, which exhibits a characteristic appearance in smears and causes superficial keratomycosis, is sensitive to voriconazole with miconazole, pimaricin, and itraconazole.
\end{abstract}

Keywords: Beauveria bassiana, keratomycosis, filamentous fungus, voriconazole, corneal infection

\section{Introduction}

Keratomycosis caused by filamentous fungi rapidly progress deep into the stroma with edematous infiltrations, often accompanied by a severe anterior chamber inflammation. In contrast, some species of filamentous fungi, such as Alternaria, can cause mild superficial corneal infections. Unfortunately, these are frequently misdiagnosed as a bacterial corneal infection (Suzuki et al 2004). Here, we report a superficial keratomycosis caused by the filamentous fungus, Beauveria bassiana (B. bassiana), and its successful treatment with voriconazole (VCZ).

\section{Case report}

An 80-year-old woman struck her left eye with the frame of her glasses. Due to continuing ocular pain and hyperemia, she was referred to our hospital 9 days after the original injury. The patient was also suffering from recurrent diabetic iritis and continuously used topical antibiotics and corticosteroids. At the time of her first visit to our hospital, there was ulceration of the corneal epithelium at the 5 o'clock region along with slight superficial infiltration, slight edema, and ciliary injection. No endothelial plaques or any immune rings were noted. Although a part of the Descemet's membrane was folded, there was only slight anterior chamber inflammation (Figure 1). A corneal scraping smear indicated the presence of Gram-positive fungal septate 


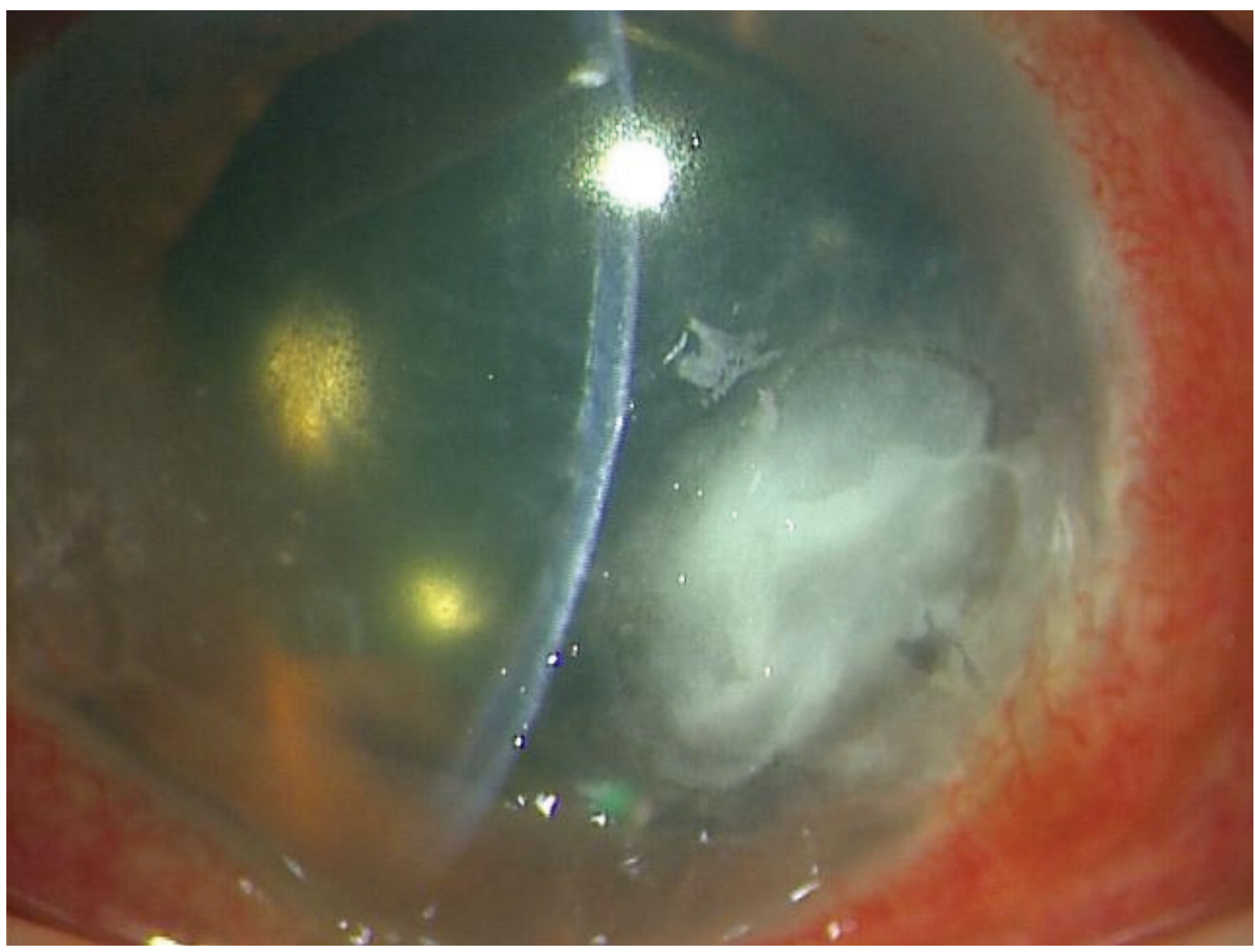

Figure I At the patient's first visit to our hospital, a slit lamp examination showed a temporal corneal ulcer at the 5 o'clock position. The lesion was restricted to the superficial stromal layer, with no endothelial plaque and very little anterior chamber inflammation.

hyphae with budding (Figure 2a), which led to a diagnosis of keratomycosis by filamentous fungus. Positive staining by Fungiflora $\mathrm{Y}$ also supported the keratomycosis diagnosis. From the culture of the corneal scraping, we were able to isolate a whitish yellow colony, with the fungus exhibiting zigzag rachis and oval conidia, which are characteristics of $B$. bassiana in slide cultures (Figure 2b). Two different microbiology laboratories, the Department of the Laboratory for Clinical Investigation at Osaka University Hospital and the Chiba University Research Center for Pathogenic
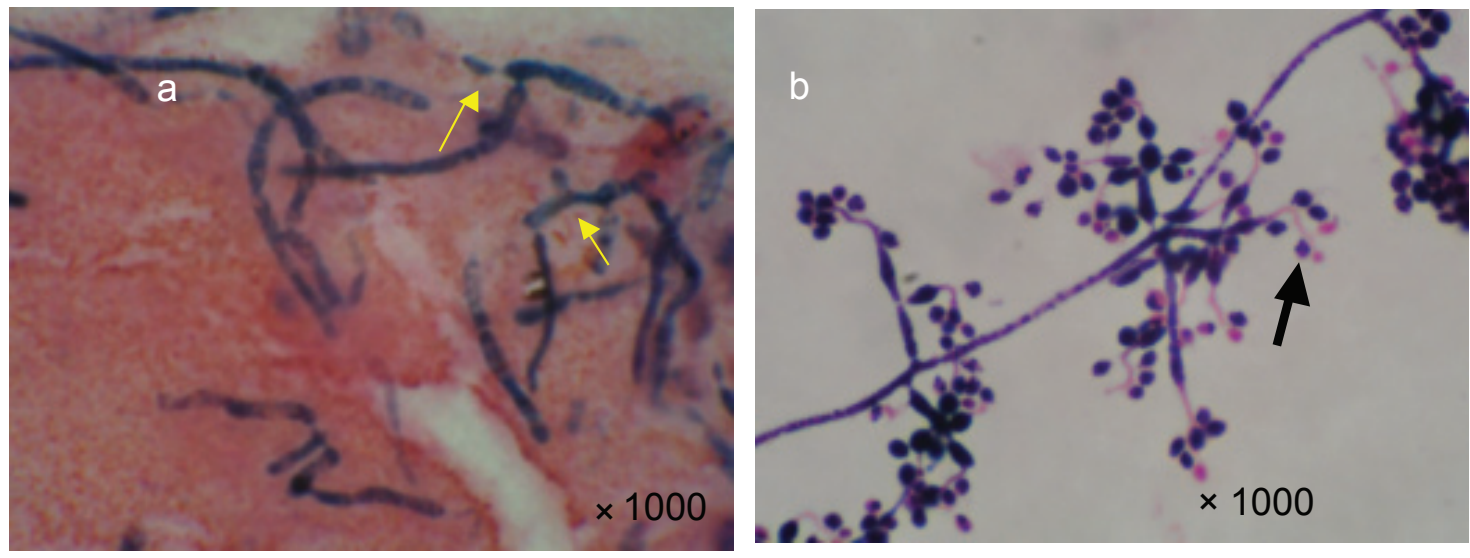

Figure 2 a Gram staining of the corneal scraping smear shows septate hyphae. Arrows indicate budding. b Beauveria bassiana cultured on a slide. Note the zig-zag rachis (arrowheads) and oval conidia. 
Fungi and Microbial Toxicoses, independently confirmed the presence of the organism. Any other organisms were detected by the culture of corneal scraping. The patient was managed with topical $0.1 \%$ miconazole (MCZ), 1\% VCZ once an hour, and $1 \%$ pimaricin (PMR) ointment administered once a day along with oral itraconazole (ITCZ: $100 \mathrm{mg} /$ day). Although there was gradual amelioration of the lesion, the presence of a band-shaped calcium deposit interrupted the re-epithelization, requiring the deposit to be scraped off on two different occasions during the healing process. The lesion healed with only mild scarring remaining after two months. In order to determine the minimum inhibitory concentration (MIC) required for each of the antimycotic agents, ASTY and E-test in vitro susceptibility tests were performed. The results for the MIC tests were: amphotericin-B: 8.0, 5-FC: 64, fluconazole (FCZ): 256, ITCZ: 0.25, MCZ: 0.5, micafangin (MCFG): 0.5, and VCZ: $0.5(\mu \mathrm{g} / \mathrm{ml})$. In addition, the organism was also found to be susceptible to topically applied antimycotic agents that included, 0.5\% VCZ, 5\% PMR, and $0.5 \% \mathrm{MCZ}$, which were administered by the disk method (Figure 3).

\section{Discussion}

This is the first report of a successful VCZ treatment of $B$. bassiana keratomycosis. VCZ is a powerful antimycotic agent that is used against filamentous fungi (Ozbek et al 2006; Sponsel et al 2006; Bunya et al 2007; Lee et al 2007; Thiel et al 2007). Although treatments of five cases of Beauveria corneal infection, four cases of B. bassiana, and one case of $B$. alba have been previously reported, for most of these patients, they also required an additional surgical procedure, such as penetrating keratoplasty or keratectomy, to completely manage the keratomycosis (Ishibashi et al 1984; McDonnell et al 1984; Sachs et al 1985; Low et al 1997; Kisla et al 2000). In order for a successful treatment to be performed without any additional surgical procedures, there needs to be an effective therapeutic regimen for antimycotic agents that can be applied in cases involving filamentous fungi. For example, in the present case, we administered VCZ and our data indicated that VCZ might have been the crucial factor that was responsible for the success of the treatment. We decided to confirm the sensitivity of the antimycotic agents at their topically applied concentrations,

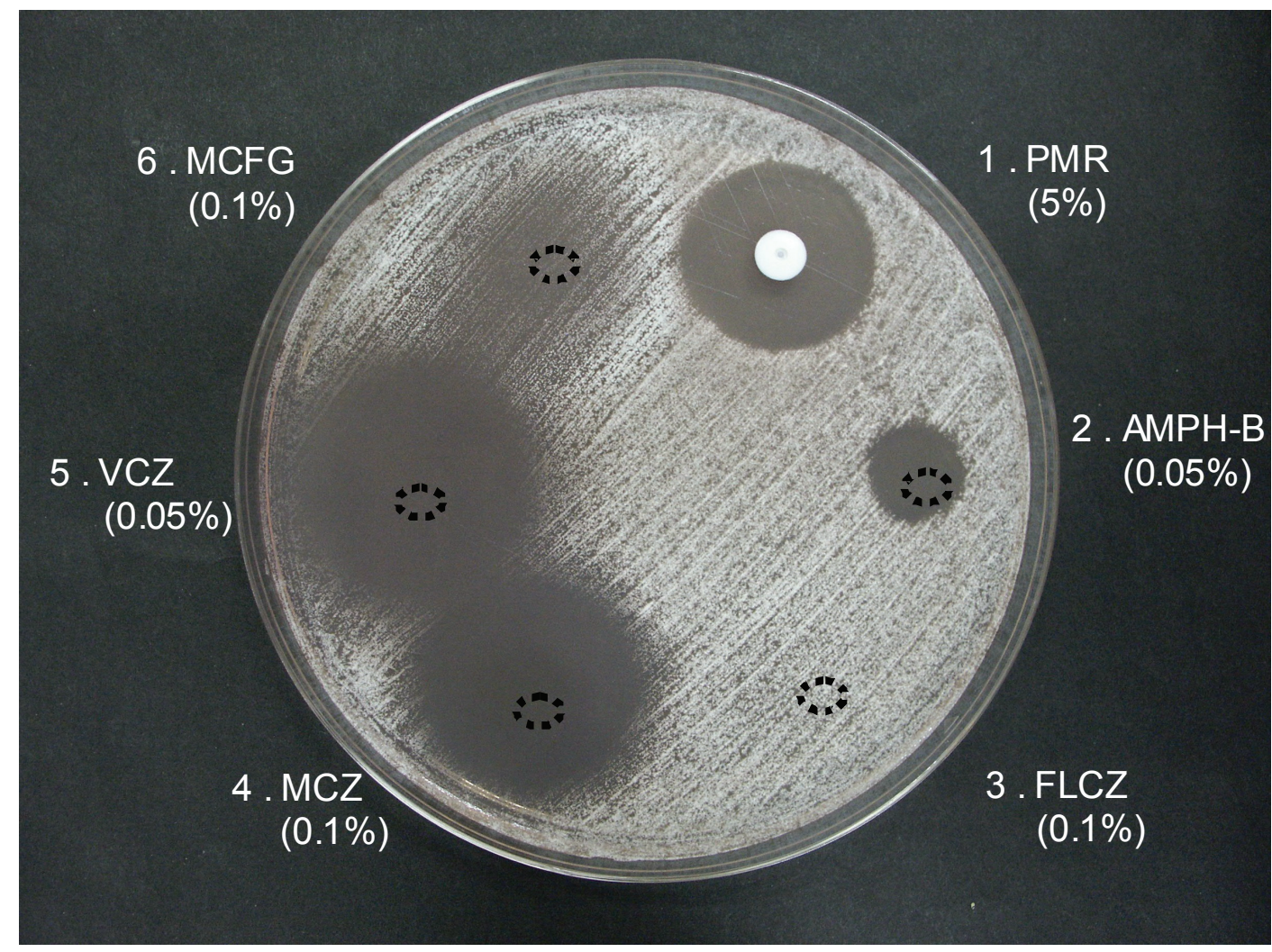

\section{: Indicates the center of the topical antimycotic drop}

Figure 3 The disk sensitivity test using topically applied antimycotic agents. A $0.05 \%$ solution of VCZ was used because the $1 \%$ VCZ caused a large inhibition circle. PMR eye drops (5\%) were used instead of ointment (I\%).VCZ, ITCZ, MCZ, and PMR all caused an inhibition circle. 
as the sensitivity judged by the CLSI standard is based upon serum concentrations, which might not always be identical to the sensitivity of the topically applied antimycotic agents. Although MCZ, PMR, ITCZ were also effective in the treatment of our patient, the result of sensitivity test using disk method indicates that VCZ was the most effective agent for B. bassiana infections.

It is also important to emphasize two characteristics of B. bassiana keratomycosis that were observed in our patient. First, we clinically noted that the keratomycosis caused by $B$. bassiana was restricted to the superficial cornea. This differs from other filamentous fungi, as the pathogenesis for $B$. bassiana is relatively weak and does not cause severe stromal infiltration or anterior chamber inflammation, as has been reported for the rabbit model (Ishibashi et al 1987). Since the lesion caused by B. bassiana extended horizontally and not vertically within the stroma, endothelial plaque and anterior inflammation were not evoked. On the contrary, Aspergillus and Fusarium, which are representative filamentous fungi of keratomycosis, will in general quickly invade deeply into the corneal stroma and induce endothelial plaque and hypopyon.

The second notable characteristic of $B$. bassiana in slide cultures is the presence of septate hyphae with budding and zigzag filaments at the apex. This characteristic appearance can be used to distinguish it from the features of Aspergillus and Fusarium. Thus, an early smear diagnosis based on these characteristics might help to ensure a successful treatment with antimycotic agents.

We were not able to determine how this organism reached the patient's eye. However, B. bassiana is found in plants and in the soil, and because this organism is entomopathogenic, it is used as a biologic for pesticides (Posada et al 2007; Safavi et al 2007) Thus, the possibility exists that while the patient was working outdoors, the frame of her eyeglasses was contaminated and upon the ocular injury that was caused by the frame of her eyeglasses, the fungi were able to invade through the damaged tissue. Our patient had a long history of recurrent diabetic iritis and continuously used topical corticosteroid. This immunocompromized situation also triggered the colonization of the fungi.
In conclusion, a weak pathogenic filamentous fungus with zig-zag rachis and budding, as determined by smear, may account for B. bassiana. VCZ proved to be a powerful tool that not only was successfully used in the current case, but also in the future might be an agent that can be used therapeutically to treat similarly infected types of patients.

\section{Acknowledgment}

We thank James Eudeikis for his English language review of our manuscript.

\section{Disclosure}

The authors report no conflicts of interest.

\section{References}

Bunya VY, Hammersmith KM, Rapuano CJ, et al. 2007. Topical and oral voriconazole in the treatment of fungal keratitis. Am J Ophthalmol, 143:151-3.

Ishibashi Y, Matsumoto Y, Takei K. 1984. The effects of intravenous miconazole on fungal keratitis. Am J Ophthalmol, 98:433-7.

Ishibashi Y, Kaufman HE, Ichinoe M, et al. 1987. The pathogenicity of Beauveria bassiana in the rabbit cornea. Mykosen, 30:115-26.

Kisla TA, Cu-Unjieng A, Sigler L, et al. 2000. Medical management of Beauveria bassiana keratitis. Cornea, 19:405-6.

Lee GA, Whitehead K, McDougall R. 2007. Management of Paecilomyces keratitis. Eye, 21:262-4.

Low CD, Badenoch PR, Coster DJ. 1997. Beauveria bassiana keratitis cured by deep lamellar dissection. Cornea, 16:698-9.

McDonnell PJ, Werblin TP, Sigler L, et al. 1985. Mycotic keratitis due to Beauveria alba. Cornea, 3:213-16.

Ozbek Z, Kang S, Sivalingam J, et al. 2006. Voriconazole in the management of Alternaria keratitis. Cornea, 25:242-4.

Posada F, Aime MC, Peterson SW, et al. 2007. Inoculation of coffee plants with the fungal entomopathogen Beauveria bassiana (Ascomycota: Hypocreales). Mycol Res, 111:748-57.

Sachs S, Baum WJ, Mies C. 1985. Beauveria bassiana keratitis. Br J Ophthalmol, 69:548-50.

Safavi SA, Shah FA, Pakdel AK, et al. 2007. Effect of nutrition on growth and virulence of the entomopathogenic fungus Beauveria bassiana. FEMS Microbiol Lett, 270:116-23.

Sponsel W, Chen N, Dang D, et al. 2006. Topical voriconazole as a novel treatment for fungal keratitis. Antimicrob Agents Chemother, 50:262-8.

Suzuki T, Uno T, Mito T, et al. 2004. Two cases of keratomycosis caused by Altenaria species. Jpn J Clin Ophthalmol, 58:65-9.

Thiel MA, Zinkernagel AS, Burhenne J, et al. 2007. Voriconazole concentration in human aqueous humor and plasma during topical or combined topical and systemic administration for fungal keratitis. Antimicrob Agents Chemother, 51:239-44. 